\title{
Lifetimes of image-potential states on copper surfaces
}

\author{
E. V. Chulkov ${ }^{1}$, I. Sarría ${ }^{2}$, V. M. Silkin ${ }^{1}$, J. M. Pitarke ${ }^{2}$, and P. M. Echenique ${ }^{1,3}$ \\ ${ }^{1}$ Materialen Fisika Saila, Kimika Fakultatea, Euskal Herriko Unibertsitatea, \\ 1072 Posta kutxatila, 20080 Donostia, Basque Country, Spain \\ ${ }^{2}$ Materia Kondentsatuaren Fisika Saila, Zientzi Fakultatea, Euskal Herriko Unibertsitatea, \\ 644 Posta kutxatila, 48080 Bilbo, Basque Country, Spain \\ ${ }^{3}$ Unidad Asociada al Instituto de Ciencia de Materiales, C.S.I.C., Cantoblanco, 28049 Madrid, Spain
}

(June 30, 2021)

The lifetime of image states, which represent a key quantity to probe the coupling of surface electronic states with the solid substrate, have been recently determined for quantum numbers $n \leq 6$ on $\mathrm{Cu}(100)$ by using time-resolved two-photon photoemission in combination with the coherent excitation of several states (U. Höfer et al, Science 277, 1480 (1997)). We here report theoretical investigations of the lifetime of image states on copper surfaces. We evaluate the lifetimes from the knowledge of the self-energy of the excited quasiparticle, which we compute within the GW approximation of many-body theory. Single-particle wave functions are obtained by solving the Schrödinger equation with a realistic one-dimensional model potential, and the screened interaction is evaluated in the random-phase approximation (RPA). Our results are in good agreement with the experimentally determined decay times.

In a variety of metal surfaces, the bulk band structure projected onto the surface presents a band gap near the vacuum level. Thus, given an electron located outside such a metal surface, it may be trapped in the vacuum well produced by the self-interaction of the electron with the polarization charge it induces in the surface [1,2]. Far from the surface, into the vacuum, this potential well approaches the long-range classical image potential, and the resulting quantized electronic states form a Rydberg-like series which converges towards the vacuum energy and is, in principle, resolvable [1]. These so-called image states were first identified experimentally [3,4] by inverse photoemission [5,6], and the first high-resolution measurements of image states were performed by the use of twophoton photoemission (2PPE) [7 10]. Combined with the use of ultrafast lasers, 2PPE has provided a powerful technique to directly probe, on a femtosecond time scale, electron dynamics of excited electrons in metals [11, 12 and, in particular, image states 12 15].

Besides their well-defined physical properties, imagepotential induced states are of general interest in surface science, because of their applicability to other areas of condensed-matter physics, providing, in particular, a very simple model to investigate the coupling of surface electronic states with the solid substrate directly in the time domain [16]. The knowledge of this coupling is cru- cial to understand many electronically induced adsorbate reactions at metal surfaces [17], and it is this coupling with the underlying substrate which governs the lifetime of the image states.

By using time-resolved 2PPE the lifetime of imagepotential states on copper surfaces has been recently determined [12 15]. Lifetimes of electrons in the $n=1 \mathrm{im}-$ age state on $\mathrm{Cu}(111)$ have been investigated [12 14], and Höfer et al [15] have used time-resolved 2PPE in combination with the coherent excitation of several quantum states to investigate the ultrafast electron dynamics of image states on the (100) surface of copper, providing an accurate experimental determination of the lifetime of the first six image states on that surface.

A quantitative evaluation of the lifetime of image states was first reported in Ref. [18], within a many-body freeelectron description of the metal surface and neglecting, therefore, any effects of the ion cores. Hydrogenic-like states with no penetration into the solid were used to describe the image-state wave functions, a step model potential was introduced to calculate the bulk final-state wave functions, simplified jellium models were used to approximate the screened Coulomb interaction, and the image-state lifetimes were evaluated, within the GW approximation of many-body theory [19], from the knowledge of the electron self-energy. More realistic imagestate wave functions were introduced in subsequent calculations 20], allowing for penetration into the crystal.

In this letter we present the results of a calculation of the lifetimes of the first three image states on a $\mathrm{Cu}(100)$ surface and the first image state on $\mathrm{Cu}(111)$, by going beyond a free-electron description of the metal surface, and including, therefore, band structure effects. First, we evaluate the electronic wave functions by solving the time-independent Schrödinger equation with a realistic one-dimensional model potential, we then use these wave functions to evaluate the screened Coulomb interaction within a well-defined many-body framework, the random-phase approximation (RPA) 21], and we finally evaluate the lifetimes from the knowledge of the imaginary part of the electron self-energy of the excited quasiparticle, which we compute within the so-called GW approximation.

As the image-state wave functions lie mainly in the vacuum side of the metal surface, and the electron moves, 
therefore, in a region with little potential variation parallel to the surface [22], we assume translational invariance in the plane of the surface, which is taken to be normal to the $z$ axis, and evaluate the damping rate of an electron in the state $\phi_{0}(z) \mathrm{e}^{\mathrm{i} \mathbf{k}_{\|} \cdot \mathbf{r}_{\|}}$with energy $E_{0}=\left(E_{0}\right)_{z}+\mathbf{k}_{\|}^{2} / 2$ (we use atomic units throughout, i.e., $e^{2}=\hbar=m_{e}=1$ ), from the knowledge of the two-dimensional Fourier transform of the electron self-energy $\Sigma\left(z, z^{\prime} ; \mathbf{k}_{\|}, E_{0}\right)$, as follows

$$
\tau^{-1}=-2 \int \mathrm{d} z \int \mathrm{d} z^{\prime} \phi_{0}^{*}(z) \operatorname{Im} \Sigma\left(z, z^{\prime} ; \mathbf{k}_{\|}, E_{0}\right) \phi_{0}\left(z^{\prime}\right) .
$$

In the GW approximation, only the first term of the expansion of the self-energy in the screened interaction is considered, and after replacing the Green function $(G)$ by the zero order approximation $\left(G^{0}\right)$, one finds:

$$
\begin{array}{r}
\operatorname{Im} \Sigma\left(z, z^{\prime} ; \mathbf{k}_{\|}, E_{0}\right)=\sum_{E_{F} \leq E_{f} \leq E_{0}} \int \frac{\mathrm{d}^{2} \mathbf{q}_{\|}}{(2 \pi)^{2}} \phi_{f}^{*}\left(z^{\prime}\right) \\
\times \operatorname{Im} W^{\text {ind }}\left(z, z^{\prime}, \mathbf{q}_{\|}, E_{0}-E_{f}\right) \phi_{f}(z)
\end{array}
$$

where the sum is extended over a complete set of final states $\phi_{f}(z) \mathrm{e}^{\mathrm{i}\left(\mathbf{k}_{\|}+\mathbf{q}_{\|}\right) \cdot \mathbf{r}_{\|}}$with energy $E_{f}=\left(E_{f}\right)_{z}+\left(\mathbf{k}_{\|}+\right.$ $\left.\mathbf{q}_{\|}\right)^{2} / 2, E_{F}$ is the Fermi energy, and $W^{\text {ind }}\left(z, z^{\prime} ; \mathbf{q}_{\|}, E\right)$ is the induced interaction:

$$
\begin{aligned}
W^{\text {ind }}\left(z, z^{\prime} ; \mathbf{q}_{\|}, E\right)= & \iint_{\times \chi\left(z_{1}, z_{2} ; \mathbf{q}_{\|}, E\right) V\left(z_{2}, z^{\prime} ; \mathbf{q}_{\|}\right) .} \mathrm{d} z_{2} V\left(z, z_{1} ; \mathbf{q}_{\|}\right)
\end{aligned}
$$

Here, $\quad V\left(z, z^{\prime} ; \mathbf{q}_{\|}\right)$and $\chi\left(z, z^{\prime} ; \mathbf{q}_{\|}, E\right)$ represent twodimensional Fourier transforms of the bare Coulomb potential and the density-response function, respectively.

In the RPA, $\chi\left(z, z^{\prime} ; \mathbf{q}_{\|}, E\right)$ satisfies the integral equation

$$
\begin{aligned}
& \chi\left(z, z^{\prime} ; \mathbf{q}_{\|}, E\right)=\chi^{0}\left(z, z^{\prime} ; \mathbf{q}_{\|}, E\right)+\int \mathrm{d} z_{1} \int \mathrm{d} z_{2} \\
& \quad \times \chi^{0}\left(z, z_{1} ; \mathbf{q}_{\|}, E\right) V\left(z_{1}, z_{2} ; \mathbf{q}_{\|}\right) \chi\left(z_{2}, z^{\prime} ; \mathbf{q}_{\|}, E\right),
\end{aligned}
$$

where $\chi^{0}\left(z_{1}, z_{2} ; \mathbf{q}_{\|}, E\right)$ represents the density-response function for non-interacting electrons. An explicit expression for $\chi^{0}\left(z_{1}, z_{2} ; \mathbf{q}_{\|}, E\right)$ can be found in Ref. [23, in terms of the eigenfunctions $\phi_{i}(z)$ of the one-electron effective hamiltonian.

We compute the image-state wave function, $\phi_{0}(z)$, and both the final states $\phi_{f}(z)$ and all the one-electron eigenfunctions involved in the evaluation of the polarizability $\chi^{0}\left(z_{1}, z_{2} ; \mathbf{q}_{\|}, E\right)$, by solving the Schrödinger equation with the one-dimensional model potential recently suggested in Ref. 24, which approaches, far outside the surface, the classical image potential and describes, inside the crystal, the self-consistent effective potential of density-functional theory. This one-dimensional potential reproduces the width and position of the energy gap at the $\bar{\Gamma}$ point $\left(\mathbf{k}_{\|}=0\right)$ and, also, the binding energies of both the $n=0$ crystal induced surface state at $\bar{\Gamma}$ and the first $(n=1)$ image-potential induced state. This model potential reproduces first-principles calculations of the wave functions and binding energies of image states in $\operatorname{Li}(100)$ 24, and also the average probability-density of the $n=1$ image state on $\mathrm{Cu}(100)$ derived by Hulbert et al 22] from a first-principles calculation (see Fig. 1). We note that the members of the Rydberg series on $\mathrm{Cu}(100)$ with quantum number $n \leq 3$ have binding energies of $0.57,0.18$ and $0.08 \mathrm{eV}$. The $n=1$ probability-density has a maximum at $3.8 \AA$ outside the crystal edge $(z=0)$, which we choose to be located half a lattice spacing beyond the last atomic layer. For the fraction of the first image state overlap probability with the bulk $(z<0)$ crystal, we find $p=0.05$. On the $\mathrm{Cu}(100)$ surface the first image state is closer to the center of the band gap than on the $\mathrm{Cu}(111)$ surface. As a result, on the latter the penetration of the first image state into the crystal is larger, $p=0.22$, and the probability-density has a maximum closer from the surface, at $2.3 \AA$ outside the crystal edge. The binding energy of this image state is $0.83 \mathrm{eV}$.

First of all, we focus on the evaluation of the damping rate of the $n=1$ image state of $\mathrm{Cu}(100)$, and we set the wave vector of the image electron parallel to the surface, $\mathbf{k}_{\|}$, equal to zero. Coupling of the image state with the crystal occurs through the penetration of the imagestate wave function into the solid and, also, through the evanescent tails of bulk states outside of the crystal. Accordingly, we have calculated separately the various contributions to the damping rate by confining the integral in Eq. (1) to either bulk $(z<0)$ or vacuum $(z>0)$ coordinates, and we have obtained the results presented in Table I. The decaying rates in the bulk are expected to be larger than in the vacuum. Also, for final-state wave vectors parallel to the surface, $\mathbf{q}_{\|}$, that are smaller than $\sqrt{2\left(E_{0}-E_{g}\right)}\left(E_{g}\right.$ represents the lower edge of the projected energy gap at the $\bar{\Gamma}$ point) there is a reduction in the phase-space because of the presence of the projected band gap, and this results in a reduced decay rate in the vacuum for which vertical transitions $\left(\mathbf{q}_{\|} \approx 0\right)$ are expected to dominate. Thus, the coupling of image states with the crystal states occurring through the bulk penetration plays an important role in the determination of the damping rate, though the penetration of the image-state wave function is small. The contribution to the damping rate coming from the interference between $z \gtrless 0$ and $z^{\prime}>0$ coordinates is comparable in magnitude and opposite in sign to both vacuum and bulk contributions, this being a consequence of the behaviour of the imaginary part of the two-dimensional Fourier transform of the self-energy [25]. The experimentally determined damping rate reported by Höfer et al 15 for the first image state on $\mathrm{Cu}(100)$, also presented in Table I, shows a reasonable agreement with our theoretical prediction, though our predicted linewidth is a little larger.

Also exhibited in Fig. 1 is the square of the 
parametrized hydrogenic-like wave function $\phi(z)=$ $\left(4 \alpha^{3}\right)^{1 / 2} z \mathrm{e}^{-\alpha z}$, with the $\alpha$ parameter chosen so as to fit the peak position of our calculated first image-state probability-density of $\mathrm{Cu}(100)$. This hydrogenic-like wave function is found to be less localized around the maximum outside the surface, and replacement of our calculated image state wave function by this approximation leads, therefore, to a damping rate (see Table I) that is too large. First, the use of this approximated initial wave function gives rise to a spurious contribution from a region just outside the surface, in which the decaying rates are noticeably larger than around the maximum, and we note that the damping rate is highly sensitive to the actual shape of the image-state wave function. Secondly, both the bulk contribution and the contribution from the interference between $z \geq 0$ and $z^{\prime}>0$ coordinates, which we have found to be of crucial importance, are completely neglected within this model.

In order to investigate the impact of band structure effects on the damping rate of image states, we have also performed a calculation in which the realistic final states $\phi_{f}(z)$ considered above are replaced by the self-consistent jellium LDA one-electron wave functions of Lang and Kohn 26], with and without the restriction that only final states with energy $\left(E_{f}\right)_{z}$ lying below the projected band gap are allowed. Both the image-state wave function and all the one-electron eigenfunctions involved in the evaluation of the screened interaction are still obtained by introducing the one-dimensional potential of Ref. [24] into the effective hamiltonian. The various contributions to the damping rate calculated in this way are presented in Table I. For $q_{\|}>\sqrt{2\left(E_{0}-E_{g}\right)}$ all final states with energy $E_{f}<E_{0}$ lie below the projected band gap; thus, the bulk and interference contributions to the damping rate remain almost unaffected by this restriction. However, as the coupling of the image state with the crystal occurring through the tails of bulk states outside of the crystal is expected to be dominated by vertical transitions $\left(\mathbf{q}_{\|} \approx 0\right)$, the vacuum contribution to the damping rate becomes dramatically smaller as final states lying within the projected band gap are not allowed.

As far as the screened interaction is concerned, systematic investigations of the role that this quantity plays in the coupling of image states with the solid have been performed [27]. We find that simplified jellium models for the electronic response lead to unrealistic results for the lifetime, though the impact of the band structure in the evaluation of the screened interaction is not large.

In a heuristic view, the bulk contribution to the decay rate for an image state might be approximated by the value of the decay rate for a bulk state at the same energy, times the fraction of the image state overlap probability with the bulk crystal. We have calculated, within the GW-RPA, the decay rate for bulk states in a homogeneous electron gas, thereby neglecting band structure effects in both the initial $\phi_{0}(z)$ and final states $\phi_{f}(z)$ and, also, in the polarizability. The damping rate for a bulk state at the energy of the first image state on $\mathrm{Cu}(100)$, times the penetration of this image state, results in a linewidth of $14 \mathrm{meV} 28$, well below the bulk contribution to our full GW-RPA surface calculation presented in Table I.

Finally, we consider the lifetime, $\tau$, of image-potential states on $\mathrm{Cu}(100)$ with quantum number $n \leq 3$, and the first image state on $\mathrm{Cu}(111)$. As before, imagestate wave functions, final states and all the eigenfunctions involved in the evaluation of the polarizability are obtained by solving the Schrödinger equation with the one-dimensional model potential of Ref. [24, and we set $\mathbf{k}_{\|}=0$. The results of these calculations are presented in Table II, together with the experimentally determined decay rates reported in Refs. 13, 14, and 15] for $\mathrm{Cu}(111)$ and $\mathrm{Cu}(100)$, respectively. We observe that both our calculated and the experimentally determined lifetimes of the image states on $\mathrm{Cu}(100)$ increase rapidly with $n$ as $\sim 1 / n^{3}$, in agreement with previous theoretical predictions [1]; this is a result of the weaker spatial overlap with the bulk that higher order image states present. As for the lifetime of the first image state on $\mathrm{Cu}(111)$ we find that it is comparable to that of $\mathrm{Cu}(100)$, though this image state on $\mathrm{Cu}(111)$ is located close to the top of the projected band gap and the wave function overlap with the bulk is, therefore, much larger. Also, the surface electronic structure of $\mathrm{Cu}(111)$ supports the well-known $n=0$ crystal-induced surface state, and we find that the decaying rate of the $n=1$ image state to this intrinsic surface state results in a linewidth of $16 \mathrm{meV}$, which represents a $40 \%$ of the total linewidth $\left(\tau^{-1}=38 \mathrm{meV}\right)$. However, both the large bulk-state overlap and the existence of the $n=0$ surface state are counterbalanced by the band gap extending on the $\mathrm{Cu}(111)$ surface below the Fermi level and the available phase space becoming, therefore, highly restricted.

In conclusion, we have reported theoretical calculations of the lifetime of image-potential states on copper surfaces, by going beyond a free-electron description of the metal surface. We have analyzed the origin and magnitude of the various contributions to the quasiparticle damping. Our results indicate that contributions to the damping rate coming from the bulk and from the interference between bulk and vacuum coordinates both play an important role in the determination of the lifetimes. We have also demonstrated that the vacuum contribution to the damping rate is highly sensitive to the location of the projected band gap. Our results are in reasonably good agreement with the experimentally determined decay times reported by Höfer et al 15 in the case of $\mathrm{Cu}(100)$, and our calculated lifetime of the first image state on $\mathrm{Cu}(111)$ is in excellent agreement with the experimental results reported in Refs. [13] and [14].

We acknowledge partial support by the Basque Hezkuntza, Unibertsitate eta Ikerketa Saila, the Spanish 
Ministerio de Educación y Cultura, and Iberdrola S.A.

[1] P. M. Echenique and J. B. Pendry, J. Phys. C 11, 2065 (1978); P. M. Echenique and J. B. Pendry, Prog. Surf. Sci. 32, 111 (1990).

[2] R. M. Osgood, Jr. and X. Wang, Solid State Phys. 51, 1 (1997).

[3] V. Dose, W. Altmann, A. Goldmann, U. Kolac, and J. Rogozik, Phys. Rev. Lett. 52, 1919 (1984).

[4] D. Straub and F. J. Himpsel, Phys. Rev. Lett. 52, 1922 (1984); D. Straub and F. J. Himpsel, Phys. Rev. B 33, 2256 (1986).

[5] J. B. Pendry, Phys. Rev. Lett. 45, 1356 (1980).

[6] P. D. Johnson and N. V. Smith, Phys. Rev. B 27, 2527 (1983).

[7] K. Giesen, F. Hage, F. J. Himpsel, H. J. Riess, and W. Steinmann, Phys. Rev. Lett. 55, 300 (1985).

[8] D. F. Padowitz, W. R. Merry, R. E. Jordan, and C. B. Harris, Phys. Rev. Lett. 69, 3583 (1992).

[9] Th. Fauster and W. Steinmann, in Electromagnetic Waves: Recent Developments in Research, Vol. 2: Photonic Probes of Surfaces, ed. P. Halevi, Elsevier, Amsterdam (1995).

[10] C. B. Harris, N.-H. Ge, R. L. Lingle jr., J. D. McNeill, and C. M. Wong, Annu. Rev. Phys. Chem. 48, 711 (1997).

[11] C. A. Schmuttenmaer et al, Phys. Rev. B 50, 8957 (1994).

[12] T. Hertel, E. Knoesel, M. Wolf, and G. Ertl, Phys. Rev. Lett. 76, 535 (1996).

[13] M. Wolf, E. Knoesel, and T. Hertel, Phys. Rev. B 54 R5292 (1996); M. Wolf, Surf. Sci. 377-379, 343 (1997).

[14] U. Höfer et al (private communication).

[15] U. Höfer, I. L. Shumay, Ch. Reub, U. Thomann, W. Wallauer, and Th. Fauster, Science 277, 1480 (1997).

[16] D. Menzel and R. Gomer, J. Chem. Phys. 40, 1164 (1964).

[17] W. Plummer, Science 277, 1447 (1997).

[18] P. M. Echenique, F. Flores, and F. Sols, Phys. Rev. Lett. 55, 2348 (1985).

[19] L. Hedin and S. Lundqvist, Solid State Phys. 23, 1 (1969).

[20] P. L. de Andrés, P. M. Echenique, and F. Flores, Phys. Rev. B 39, 10356 (1989).

[21] See, e.g., D. Pines, Elementary excitations in solids, Chapter 3 (Addison Wesley, New York, 1963).

[22] S. L. Hulbert, P. D. Johnson, M. Weinert, and R. F. Garret, Phys. Rev. B 33, 760 (1986).

[23] A. G. Eguiluz, Phys. Rev. Lett. 51, 1907 (1983).

[24] E. V. Chulkov, V. M. Silkin, and P. M. Echenique, Surf. Sci. 391, L1217 (1997).

[25] J. J. Deisz, A. G. Eguiluz, and W. Hanke, Phys. Rev. Lett. 71, 2793 (1993).

[26] N. D. Lang and W. Kohn, Phys. Rev. B 1, 4555 (1970).

[27] I. J. Sarría, E. V. Chulkov, V. M. Silkin, J. M. Pitarke, P. M. Echenique, and A. G. Eguiluz (unpublished).

[28] In the high-density limit and for electron energies $(E)$ just above the Fermi level $\left(E_{F}\right)$ the damping rate is found to be (see J. J. Quinn and R. A. Ferrell, Phys. Rev. 112, $812(1958)) \tau^{-1}=0.00251 r_{s}^{5 / 2}\left(E-E_{F}\right)^{2} \mathrm{eV}^{-1}$, in linewidth units. This approximated formula gives for the damping rate for a bulk state at the energy of the first image state on $\mathrm{Cu}(100)$, times the penetration, a linewidth of $24 \mathrm{meV}$.

TABLE I. Damping rates, in linewidth units $(\mathrm{meV})$, of the $n=1$ image state on $\mathrm{Cu}(100)$. (a) Our full calculation, as described in the text, together with the experimental damping rate of Ref. 15. (b) The result of our calculation when our realistic image-state wave function is replaced by the hydrogenic-like wave function described in the text. (c) and (d) The result of our calculation when our realistic final states are replaced by the self-consistent jellium LDA wave functions of Lang and Kohn 26], (c) with and (d) without the restriction that only final states with energy $\left(E_{f}\right)_{z}$ lying below the projected band gap are allowed.

\begin{tabular}{lccccc}
\hline \hline & Bulk & Vacuum & Interference & Total & Experiment \\
\hline (a) & 24 & 14 & -16 & 22 & 16.5 \\
(b) & - & 71 & - & 71 & \\
(c) & 19.5 & 13 & -11 & 21.5 & \\
(d) & 21 & 58 & -12 & 67 & \\
\hline \hline
\end{tabular}

TABLE II. Calculated lifetimes, in femtoseconds, of the $n \leq 3$ image states on $\mathrm{Cu}(100)$ and the first image state on $\mathrm{Cu}(111)$, together with the experimentally determined lifetimes of Refs. 13,14 and 15 for $\mathrm{Cu}(111)$ and $\mathrm{Cu}(100)$, respectively. The damping rate of Eq. (1) is related to the lifetime, $\tau$, by $\tau^{-1} \tau=1$ a.u. $=660 \mathrm{meV}$ fs.

\begin{tabular}{|c|c|c|c|c|}
\hline & & $\mathrm{Cu}(100)$ & & $\mathrm{Cu}(111)$ \\
\hline & Theory & Experiment & 15] Theory & Experiment 13,14 \\
\hline$n=1$ & 30 & $40 \pm 6$ & 17.5 & \begin{tabular}{l|l|}
$18 \pm 5$ & 13 \\
$15 \pm 5$ & 14
\end{tabular} \\
\hline$n=2$ & 132 & $110 \pm 10$ & & \\
\hline$n=3$ & 367 & $300 \pm 15$ & & \\
\hline
\end{tabular}

FIG. 1. Probability-density of the $n=1$ image state on $\mathrm{Cu}(100)$ (solid line) and the hydrogenic-like approximation described in the text (dashed line). Stars represent the probability-density of the $n=1$ image state, averaged parallel to the surface, as reported in Ref. [22. 


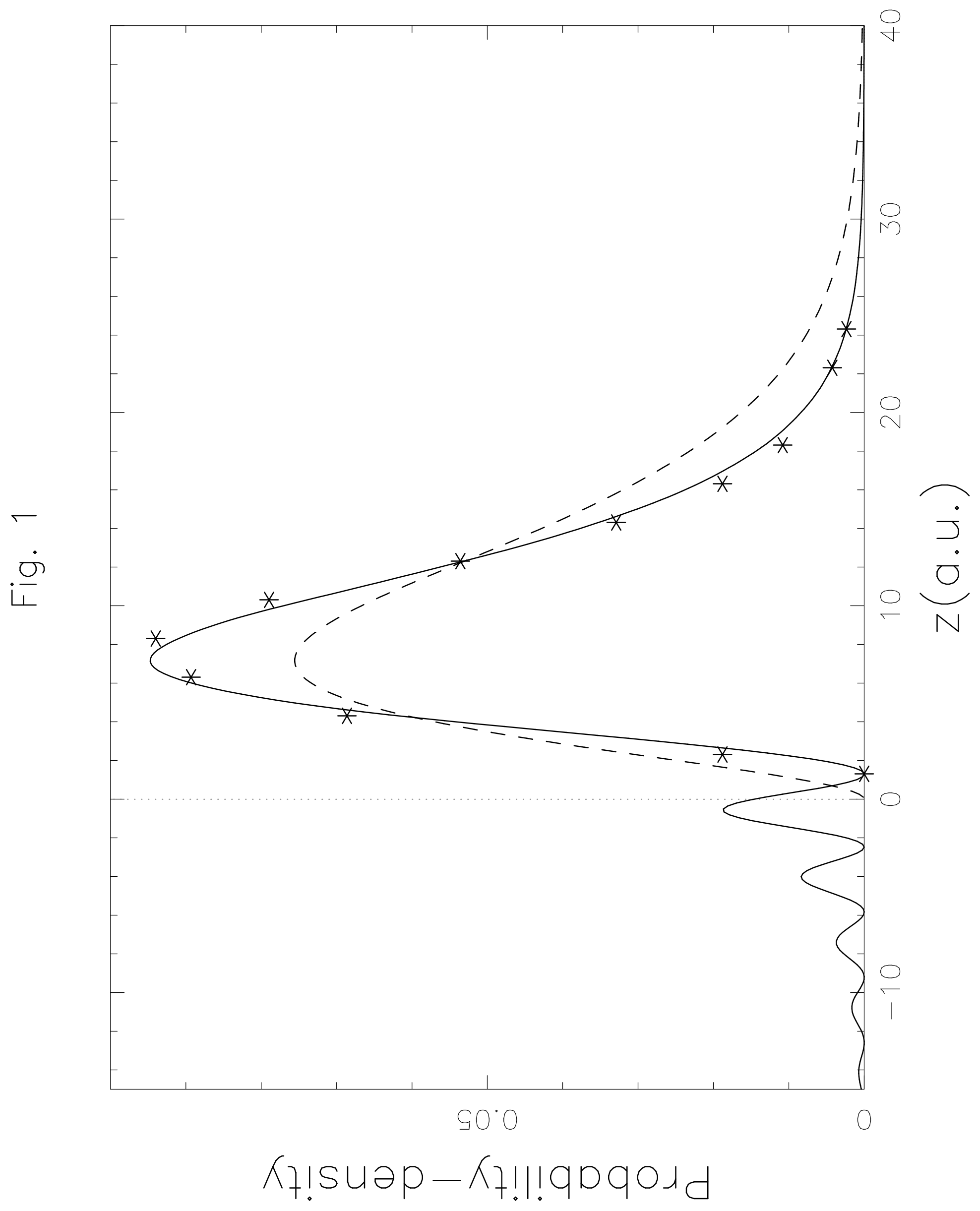

